Elsevier

PAI 01139

\title{
Pain measurement in reflex sympathetic dystrophy syndrome
}

\author{
Gary Davidoff, Kathy Morey, Mary Amann and Janice Stamps \\ Department of Physical Medicine and Rehabilitation, University of Michigan Medical Center, Ann Arbor, MI 48109 -0042 (U.S.A.)
}

(Received 6 April 1987, revised received and accepted 9 July 1987)

\begin{abstract}
Summary The reflex sympathetic dystrophy syndrome (RSDS) consists of a triad of pain, edema and sympathetic dysfunction of an extremity following trauma, peripheral nerve injury or central nervous system disorder. Reflex sympathetic dystrophy syndrome is a difficult and costly pain syndrome to treat. One of the difficulties in evaluating treatment efficacy is the objectification and quantification of patient findings. The purpose of this investigation was to develop a simple, reproducible and systematic evaluation of pain and neurologic impairment in RSDS. The protocol evaluation included the following measures of pain and impairment: (1) objectification of distal joint pain by palpation ( $0-4$ scale); (2) volumetric measurement by water displacement of the distal limb; ( 3 ) skin temperature measurement; (4) active range of motion assessment of the affected limb (AROM); (5) McGill Pain Questionnaire (MPQ); and (6) visual analogue pain scale (VAS).

Seventeen subjects with definite or probable RSDS were evaluated. The mean age of patients was 35.6 years \pm 8.0 years ( \pm 2 S.E.M.), and the mean duration of symptoms was $11.8 \pm 4.8$ months. There were an equal number of subjects with upper and lower extremity involvement. The VAS had significant correlations with limb volume, AROM-lower extremity and joint pain indices $(P<0.0005)$. The MPQ subscales had robust correlation with the joint pain indices alone $(P<0.0001)$. Skin temperature was not predictive of changes in joint pain score, AROM, limb volume or subjective pain measures. However, there was internal consistency between volumetric and AROM measures and joint pain indices. This protocol is recommended for use in therapeutic trials for the treatment of the RSDS.
\end{abstract}

Key words: Reflex sympathetic dystrophy; Pain measurement

\section{Introduction}

The reflex sympathetic dystrophy syndrome (RSDS) consists of a triad of pain, edema and vasomotor instability of an extremity following peripheral limb trauma or central nervous system disorder [12-14]. In this study, we were interested in patients who met Kozin's criteria for definite or probable RSDS [12-14]. This included distal limb pain, and either diffuse swelling or vasomotor instability of the extremity. The RSDS is an extremely difficult and costly syndrome to treat.

Correspondence to: Gary Davidoff, M.D., Department of Physical Medicine and Rehabilitation, 1D204H University Hospital, Box 0042, Ann Arbor, MI 48109-0042, U.S.A.
One of the difficulties in previous reports of treatment efficacy is the lack of uniform quantification of treatment outcome, as defined by change in pain measurement or improvement in function. The non-uniformity of outcome measurement, as well as the lack of consistent diagnostic criteria in previous studies [1,2,4,5,7,11-14,17,18,20,22], makes it difficult to draw inferences from previous therapeutic trials in RSDS patients. We have developed a simple, quick and reproducible method for systematic evaluation of pain and impairment in the RSDS. The purpose of this study was to investigate the utility of this protocol and how various objective and subjective measurements of pain and function varied with the course of treatment in this patient group. 


\section{Methods}

\section{Subjects}

Seventeen patient-subjects were chosen for participation and measurement in this study. Inclusion criteria consisted of the following: (A) age over 11 years; (B) duration of pain syndrome $>1$ month; $(\mathrm{C})$ no evidence of involvement of a contralateral extremity, as defined by a negative clinical exam and a negative 3-phase bone scan; (D) no previous history of sympathectomy of the affected extremity. All patients met Kozin's criteria for definite or probable RSDS [12-14]. This included distal limb pain and either diffuse limb edema or vasomotor instability of the extremity (e.g., abnormal skin temperature, trophic skin changes, hyperhydrosis).

\section{Procedure}

Each patient underwent a comprehensive interview in which the history of the RSDS was reviewed in detail. Pertinent data were obtained including age, sex, duration of pain, presumed etiology, previous diagnostic and therapeutic interventions and associated medical conditions. A thorough neurologic and general physical examination was performed on each patient. A 3phase bone scan with $\mathrm{Tc}-99 \mathrm{~m}(\mathrm{Sn})$ methylenediphosphonate (Tc-99m MDP) was performed.

At the initial consultation the following evaluation was performed by research physical or occupational therapists. Members of the staff of the physical therapy division evaluated patients with lower extremity RSDS and the upper extremity patients were evaluated by members of the occupational therapy division. Two of the authors (K.M., M.A.) assured consistent evaluation between a small cadre of treating therapists. The evaluation protocol consisted of the following:

(1) Measurement of joint pain of the hand or foot by palpation using a 4-point scale as follows: $0=$ no pain; 1 - mild pain to deep palpation; $2=$ severe pain to deep palpation; $3=$ severe pain to mild palpation; $4=$ hyperesthesia.

Joint pain was evaluated in the hand and wrist for all interphalangeal, metacarpalphalangeal and wrist joints. Evaluation of the foot and ankle consisted of palpation of the interphalangeal and metatarsalphalangeal joints of the foot and the tibio-talar joint. A total joint pain score was derived by summation of the individual pain scores for each joint.

(2) Limb volume. Evaluation of asymmetric increase in limb volume was performed by volumetric measurement of the extremities. For measurement of the upper extremity, the hand was immersed to a point just proximal to the lateral styloid process of the wrist in a 2 liter laboratory beaker. Measurement of the foot was performed by immersing the foot into a 5 gallon glass vessel (fish tank). The foot was immersed to a point $6 \mathrm{~cm}$ proximal to the distal edge of the lateral malleolus. In both cases the volume measurement was determined based on the average of 3 trials. The contralateral unaffected extremity was also measured to serve as a control for each patient. The percent limb volume (LIMB VOL) increase was defined as:

$\frac{\left(\text { volume }_{\text {limb affected }}-\text { volume }_{\text {limb unaffected }}\right) \times 100}{\text { volume }_{\text {limb unaffected }}}$.

A limb volume difference $>5 \%$ was considered abnormal.

(3) Skin temperature measurement of hands or feet (in ${ }^{\circ} \mathrm{C}$ ). Skin temperature of the hand was measured over the palmar surface in the web space between the first and second metacarpal joints and fourth and fifth metacarpal joints. Lower extremity temperatures were taken on the dorsum of the foot between the first and second metatarsal joints and between the fourth and fifth metatarsal joints. These temperatures were averaged and subtracted from the mean temperature obtained in similar fashion from the unaffected contralateral extremity. A temperature difference of $\pm 0.5^{\circ} \mathrm{C}$ was considered abnormal.

(4) Active range of motion (AROM) of the affected distal extremity. In the upper extremity this was generated by the average distance from the digital pads (II-V) to the distal palmar crease. For the lower extremity this consisted of goniometric measurement of ankle dorsiflexion, plantar flexion, eversion and inversion. The degree of impairment of active range of motion of the lower extremity (AROM-LE) was given by the difference 
between the expected degrees of normal ankle range minus the actual measurement of the active range of motion of the ankle in the planes of eversion, inversion, plantar and dorsiflexion.

Prior to the actual physical assessment, the following subjective pain inventories were administered to each patient:

(A) The McGill Pain Questionnaire (MPQ). The MPQ [15] generates information concerning affective, sensory and evaluative aspects of pain. Two types of measurement were obtained from the MPQ: the pain rating index (PRI) was based upon the rank values of words selected from a 20 -item inventory to describe the sensory (PRI-S), affective (PRI-A), evaluative (PRI-E), and total (PRI-T) aspects of pain. In addition the number of words chosen (NWC) from the same 20 category word list was recorded. The MPQ has been reported by many authors to be a valid and reliable instrument for pain assessment $[8,9,16,19,21]$. In this study the patient was allowed to fill out the MPQ while an investigator was avallable to answer questions concerning directions for administration. No information was given by the investigator with regard to definition of various pain items.

(B) The Visual Analog Scale [10]. This was a $100 \mathrm{~mm}$ line printed on a simple $8.5^{\prime \prime} \times 11^{\prime \prime}$ sheet of paper. One extreme of the line indicates no pain, the other extreme indicates severe pain. The patient was asked to place an $\mathrm{x}$ where he/she would rank his/her present pain intensity. The VAS has been demonstrated to be a reliable and reproducible evaluation of pain intensity for chronic pain patients [10].

This entire protocol was repeated at bi-weekly intervals for an 8 week period of treatment for each patient.

\section{Data analysis}

Comparisons of parametric variables including pain duration and pain measurement between upper and lower extremity patient groups were performed using a Student's $t$ test. All confidence intervals are given as \pm 2 standard errors of the mean (2 S.E.M.). Simple and multiple linear regression analyses were used to evaluate the relationship between objective and subjective pain measures observed during various intervals over the course of treatment. All data analyses were performed utilizing the Michigan Interactive Data Analysis System [6].

\section{Results}

Seventeen subjects with definite or probable RSDS [12-14] were evaluated. The mean age was $35.6 \pm 8.0$ years $( \pm 2$ S.E.M.). Five patients $(29.4 \%)$ were male. Nine patients $(52.9 \%)$ had upper extremity involvement, with the remaining patients having solely lower extremity involvement. Preceding trauma could be implicated as an etiologic factor in 14 patients $(82.4 \%$ ), with a latency of weeks to months between the predisposing factor and the development of symptoms consistent with RSDS. Two patients had a history of severe central nervous system damage (stroke, meningitis) and 1 patient had no attributable factor preceding the development of symptoms. The mean duration of time between the onset of symptoms and evaluation in clinic was $11.8 \pm 4.8$ months.

Fifteen patients underwent electrodiagnostic studies (EDX) performed by a physiatrist or neurologist in the Electrodiagnostic Laboratory of The University of Michigan Hospitals. Twelve EDX studies were normal. The remaining 3 abnormal studies consisted of mononeuropathy [2] and polyradiculopathy [1]. All patients underwent a 3-phase bone scan. Nine patients had abnormally increased uptake in the delayed images consistent with RSDS [12-14]. The remaining patients had normal blood flow, blood pool and uptake. All patients had evidence of articular tenderness in the hand and wrist, or foot and ankle. In the hand, the metacarpal joint was most commonly affected. In the foot, the most commonly involved joints were the metatarsals. Nine patients had abnormally increased limb volume. Twelve patients had abnormally decreased skin temperature $\left(>0.5^{\circ} \mathrm{C}\right.$ difference between extremities). Eleven patients had evidence of trophic skin changes and 4 patients had evidence of abnormal sweating.

All patients were treated with a therapeutic exercise program for an 8 week period (biw or tiw). Most patients also received oral corti- 
costeroids or regional sympathetic blockade as adjunctive therapy. The purpose of this study was to develop a useful clinical instrument for measurement of outcome in the RSDS. Consequently, data are not reported stratified by treatment method.

The baseline pain rating index (PRI) from the McGill Pain Questionnaire (MPQ) was $23.1 \pm 6.5$. The number of words chosen (NWC) from the MPQ at baseline was $9.6 \pm 1.9$. The baseline visual analog scale (VAS) rating was $55.1 \pm 13.0$.

Squared correlation coefficients from linear regression analyses of pain and function measures before and during treatment are given in Table I. The VAS had significant correlations with limb volume, active range of motion and joint pain (Fig. 1). The PRI-T and NWC correlated only with active range of motion of lower extremities and joint pain indices. There was also a very strong correlation with active range of motion of upper or lower extremities and limb volume. There was no correlation whatsoever between changes in skin temperature and either objective or subjective

\section{TABLE 1}

SQUARED CORRELATION COEFFICIENTS FOR LINEAR REGRESSION OF PAIN AND FUNCTION MEASURES IN REFLEX SYMPATHETIC DYSTROPHY SYNDROME

AROM $=$ active range of motion; NWC = number of words chosen-MPQ: $\mathrm{PRI}=$ pain rating intensity-MPQ; VAS = visual analog pain scale; PRI-T = pain ranking index, total.

\begin{tabular}{|c|c|c|c|c|c|}
\hline & $\begin{array}{l}\text { Limb } \\
\text { vol. }\end{array}$ & $\begin{array}{l}\text { Skin } \\
\text { temp. }\end{array}$ & $\begin{array}{l}\text { AROM- } \\
\text { UE }\end{array}$ & $\begin{array}{l}\text { AROM- } \\
\text { LE }\end{array}$ & $\begin{array}{l}\text { Joint } \\
\text { pain }\end{array}$ \\
\hline Limb vol. & - & & & & \\
\hline Skin temp. & 0.049 & - & & & \\
\hline AROM-UE & $0.180^{a}$ & 0.111 & - & & \\
\hline AROM-LE & $0.454^{f}$ & 0.022 & - & - & \\
\hline Joint pain & $0.246^{f}$ & 0.030 & 0.068 & $0.636^{\mathrm{d}}$ & - \\
\hline NWC & 0.011 & 0.009 & 0.026 & $0.159^{a}$ & $0.278^{f}$ \\
\hline VAS & $0.160^{\mathrm{e}}$ & 0.000 & $0.167^{\circ}$ & $0.508^{f}$ & $0.341^{f}$ \\
\hline PRI-T & 0.023 & 0.011 & 0.053 & $0.263^{c}$ & $0.324^{\mathrm{f}}$ \\
\hline
\end{tabular}

a $P<0.05$.

b $P<0.01$.

c $P<0.005$.

d $P<0.001$.

$\because P<0.0005$.

f $P<0.0001$.

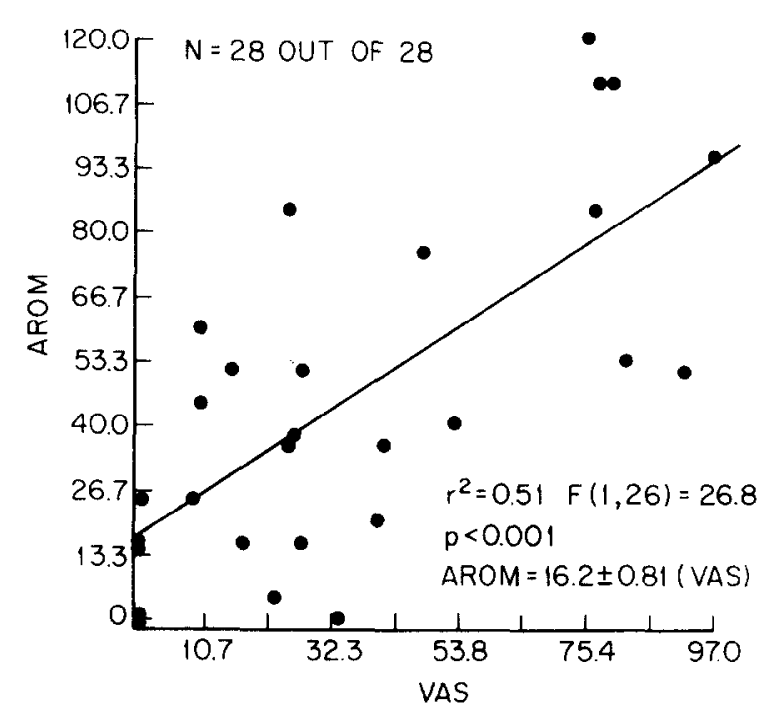

Fig. 1. Active range of motion (AROM) versus visual analog scale (VAS), lower extremities only.

measures of pain and function in this patient group.

The squared correlation coefficients for linear regression analyses of pain scales and subscales before and during treatment are reported in Table II. There was a robust correlation between the PRI subscales, PRI-T and NWC of the MPQ (Fig.

TABLE II

SQUARED CORRELATION COEFFICIENTS FOR LINEAR REGRESSION OF PAIN SCALES AND SUBSCALES IN REFLEX SYMPATHETIC DYSTROPHY SYNDROME

$\mathrm{NWC}=$ number of words chosen; $\mathrm{VAS}=$ visual analog scale; PRI-T = pain ranking index, total; PRI-A = pain ranking index, affective; PRI-E = pain ranking index, evaluative; PRI-S - pain ranking index, sensory.

\begin{tabular}{lllllll}
\hline & NWC & VAS & PRI-T & PRI-A & PRI-E & PRI-S \\
\hline NWC & - & & & & & \\
VAS & $0.368^{\mathrm{f}}$ & - & & & & \\
PRI-T & $0.822^{\mathrm{f}}$ & $0.445^{\mathrm{f}}$ & & & & \\
PRI-A & $0.29^{\mathrm{f}}$ & $0.05^{\mathrm{f}}$ & $0.35^{\mathrm{f}}$ & - & & \\
PRI-E & $0.42^{\mathrm{f}}$ & $0.25^{\mathrm{c}}$ & 0.53 & 0.33 & - & \\
PRI-S & $0.73^{\mathrm{f}}$ & $0.51^{\mathrm{f}}$ & 0.83 & $0.34^{\mathrm{f}}$ & $0.43^{\mathrm{f}}$ & - \\
\hline
\end{tabular}

: $P<0.05$.

b $P<0.01$.

$P<0.005$.

${ }^{4} P<0.001$. 


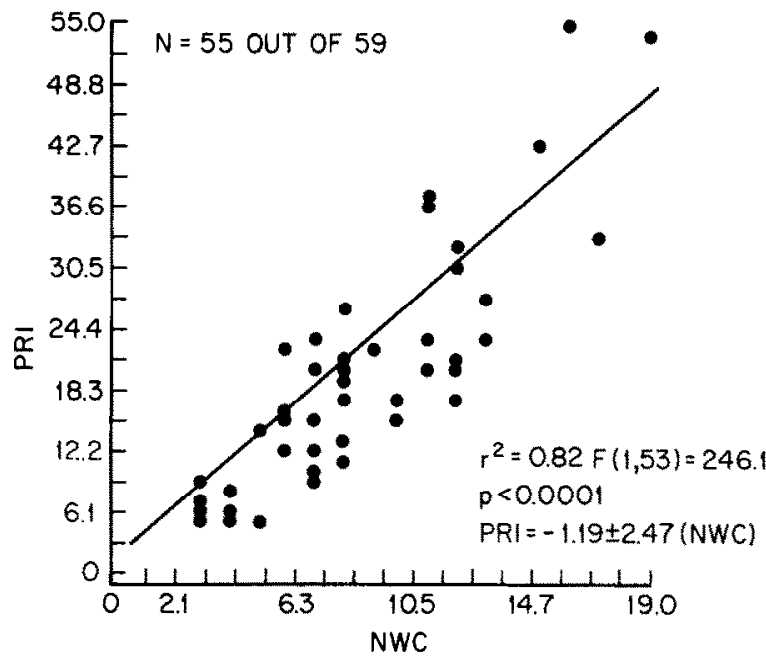

Fig. 2. Pain ranking index (PRI) versus number of words chosen (NWC).

2). There was a somewhat less robust correlation between PRI-T, affective pain rate index (PRI-A), sensory pain rating index (PRI-S) and the VAS. There was no correlation between the PRI-A subscale and the VAS.

The following case illustrates how this method can be used to chart patient progress during treatment. F.R. was a 16-year-old right-handed white male who presented to the Emergency Room of the University of Michigan Hospital in April, 1986. There was a history of mild discomfort in the right hand and wrist since October, 1985. At that time he had suffered trivial injuries in football practice to the right shoulder without fracture or dislocation. The patient described swelling and discoloration of the extremity off and on over subsequent months. Approximately 3 weeks prior to presentation, he developed severe swelling and pain in the right hand and increased numbness distal to the mid-forearm in a glove-like distribution. Electrodiagnostic studies were negative. He also underwent non-invasive flow studies and venography which were negative for venous thrombotic disease of the right upper extremity. The patient was then given the diagnosis of definite reflex sympathetic dystrophy of the right upper extremity. At the initial presentation, the right hand was increased $22 \%$ beyond the volume of the left hand. There was no skin temperature difference. Active range of motion was profoundly impaired, with an average measurement of $4.9 \mathrm{~cm}$. The objective joint pain score was 16 . The Melzack Pain Questionnaire demonstrated 7 words chosen with a pain rating index (PRI) of 12 . The visual analog scale score was 48 . The patient was not taking any medications at the time of presentation. Following initial clinical assessment, the patient was placed on a 2 week course of oral prednisone, $1 \mathrm{mg} / \mathrm{kg}$. This was followed by a 2 week taper of oral corticosteroid. In addition, the patient began an aggressive physical and occupational therapy program twice a week. Fig. 3 il-

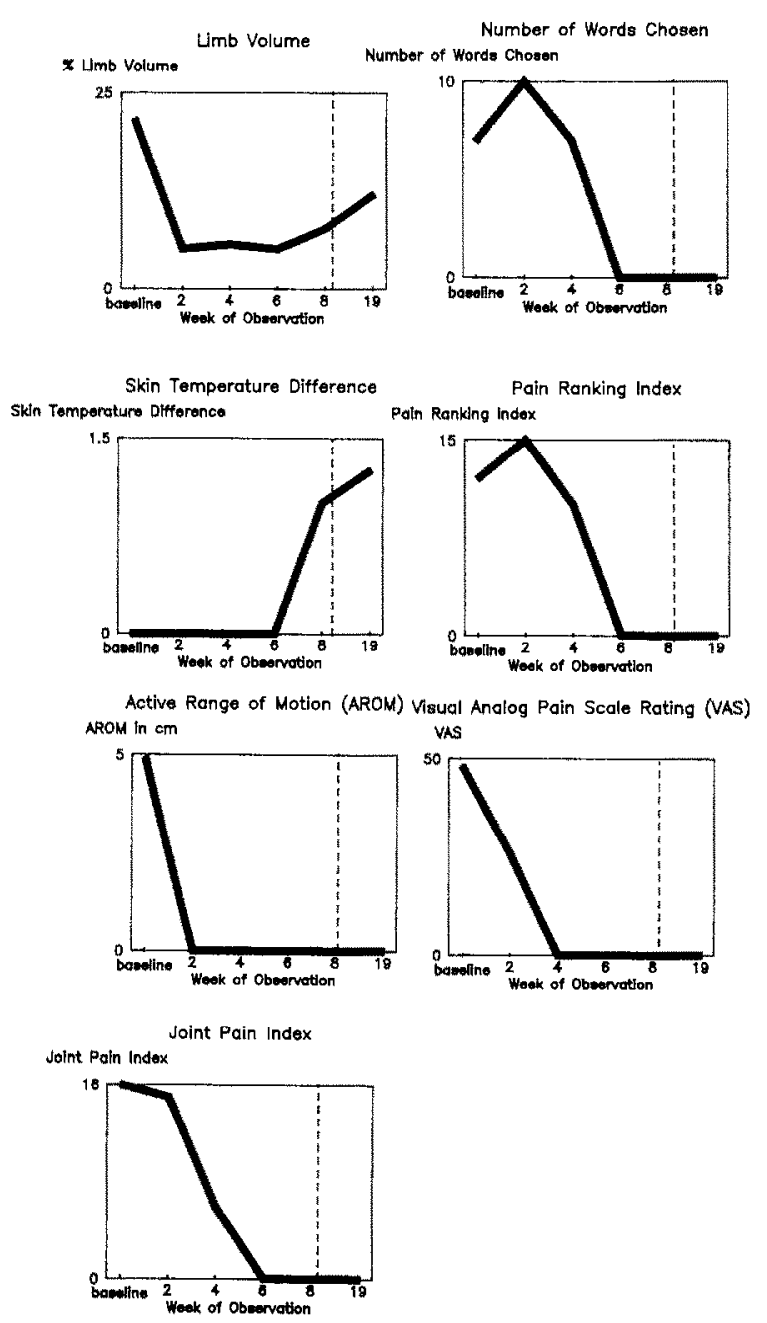

Fig. 3. Pain and function measures during and after treatment of patient F.R. for reflex sympathetic dystrophy. 
Iustrates the patient's progress, monitored by serial 2 week evaluations. By week 8 the patient had nearly no edema of the right hand and no evidence of objective or subjective pain in the right hand. At assessment 19 weeks after the beginning of treatment, the patient had developed some edema of the right hand with a $1.25^{\circ} \mathrm{C}$ skin temperature difference, with the right hand cooler than the left. The patient continued to deny any objective or subjective evidence of pain. However, it was felt on this basis that the patient continued to have abnormal sympathetic flow to the right upper extremity and was cautioned against participating in contact sports or other activities which might be likely to traumatize his right arm and reproduce the syndrome.

\section{Discussion}

Reproducible and reliable outcome measurements are critical to efficacy studies of treatment outcome in the RSDS. Wang et al. [22] conducted an open trial of central sympathetic blockade for the treatment of the RSDS. The comparison group consisted of a historical patient cohort which had received only physical therapy. Their outcome measure was a 5-point rating of subjective pain relief. Measurement of limb edema, joint pain, vasomotor instability or function was never documented either before or after treatment. These measures would have done much to support the validity of their findings. Similar difficulties in design of outcome measurement can be found in other studies $[4,11,17,20]$.

Bonelli et al. [1] quantified vasomotor changes, range of motion (not specified if this was active or passive), visual analog pain scale and presence of hyperpathia in their trial comparing the efficacy of regional versus central sympathetic blockade for the treatment of the RSDS. However, these outcomes were reported as either 'improvement' or 'no change.' No statistical analysis was performed to analyze the group changes on these variables after treatment. Poplawski et al. [18] truncated similar detailed findings of objective and subjective pain and function in their trial of regional sympathetic block into a 5-point scale reflecting overall change in pain, function and edema. No description was given as to how this scale was generated or what cut-off points were used for the collapsed rating of pain and function measures. A similar problem in design methodology can be demonstrated in the study by Driessen et al. [5].

Repeated measures of pain and function were reported in the trials by Glynn et al. [7] and Kozin et al. [12-14]. Glynn et al. [7] described repeated measures taken at 3 points: pre-sympathetic hlockade, immediately after block and 7 days post-sympathetic blockade. Follow-up time was inadequate to allow observation of improvement for a clinically relevant and functional period (e.g., 3 -6 months). Kozin et al. [12-14] performed systematic repeated measures of ring size (indirect measure of edema), joint tenderness (via dolorimetry) and grip strength in their clinical trials of oral corticosteroids and stellate ganglionic blockade. Ring size measurement has not seen great clinical application, because of the multiple measurement required (all interphalangeal joints on both hands). In addition, ring size measurement is more difficult to reproduce in the foot, due to the smaller circumference of the digits. Grip strength is a poor measure of functional outcome in a patient sample with traumatic peripheral mononeuropathies and central nervous system disorders, common precipitants of the RSDS [4,20].

The purpose of this investigation was to develop a protocol of objective and subjective measures of pain and function to evaluate outcome in the reflex sympathetic dystrophy syndrome (RSDS). The objective measurements of limb volume, skin temperature, and active range of motion were reproducible within $\pm 5 \%$ on replicate observations on the same patient on the same day. The time required to perform the evaluation was approximately $45 \mathrm{~min}$. The criteria for inclusion in this study were strictly enforced. Only patients with definite or probable RSDS were included to ensure a homogenous patient sample. Patients were excluded if they had bilateral RSDS, as the contralateral extremity was used as a control for evaluating differences in skin temperature and limb volume. Consequently, this protocol would not be applicable for patients with bilateral 
findings consistent with RSDS. Patients with surgical sympathectomy or chemical sympathectomy were excluded, since these patients would have had abnormal skin temperature which would be unlikely to change with successful treatment.

Several interesting findings can be interpreted from Table I, describing the relationship between objective and subjective measures of pain and function during treatment for the RSDS. There was no correlation between skin temperature and any of the objective or subjective parameters of pain and function. Since most patients demonstrated a clear improvement in their pain syndrome during the treatment program, the continuing abnormality in skin surface temperature was suggestive of a quiescent, subclinical picture of ongoing abnormal sympathetic activity. This may explain why many patients continue to complain of recurring problems with chronic pain in RSDS. There was a strong positive correlation between active range of motion of the lower extremities and joint pain, but no such correlation was found for active range of motion of the upper extremities (Table I). Nearly all patients achieved normal active range of motion of the hand at the completion of treatment. This was apparently accomplished in spite of the continuing pain of which patients complained. The number of words chosen (NWC) and the total pain rating index (PRI-T) correlated only with active range of motion of lower extremities and joint pain indices (Table I). Multiple linear regression analyses indicated that the majority of variance for these 2 subjective pain indices (NWC, PRI-T) could be explained solely on the basis of change in the joint pain index (Table I). However, the visual analog scale (VAS) demonstrated robust and statistically significant positive correlations with all objective measures of pain and function, with the exception of skin temperature (Table I). Although the VAS is generally considered limited by its unidimensional character in pain studies, it would appear to be a potentially valuable instrument, superior to the MPQ for brief clinical assessment of patient progress during treatment for the RSDS.

Use of the MPQ provided quantification of sensory, affective and evaluative components of pain in this patient group during treatment. Val- ues of the MPQ subscales generated by this sample equaled or exceeded those reported by patients with other pain syndromes, including menses, arthritis, cancer, phantom pain, toothache and spinal cord dysesthetic pain $[3,8,9,15,16,21]$. Intercorrelation of the $3 \mathrm{MPQ}$ subscales (Table II) suggests that most of the variance in the total pain rating index (PRI-T) was the result of the number of words chosen (NWC) and the sensory pain rating index (PRI-S). There was a robust correlation between PRI-T, PRI-S, evaluative pain rating index (PRI-E) and the visual analog scale (VAS) (Table II). There was no correlation between PRI$A$ and the VAS, which is consistent with the unidimensional characteristics of pain description generated by the VAS [10].

This investigation describes a simple, reproducible and internally consistent protocol for systematic measurement of pain function in the RSDS. A uniform system of outcome measurement will aid in allowing comparison of various clinical trials for the treatment of this painful and disabling condition.

\section{Acknowledgements}

Funding for this project was provided by a Clinical Investigator Development Award (G.D.) from the National Institute of Neurological and Communicative Disorders and Stroke, Bethesda, MD (NS01120-01).

Secretarial support was provided by Jayne Long.

\section{References}

1 Bonelli, S., Conoscente, F., Movilia, P.G., Rostelli, L., Francucci, B. and Grossi, E., Regional intravenous guanethidine versus stellate ganglion blocks in reflex sympathetic dystrophy: a randomized trial, Pain, 16 (1983) 297-307.

2 Cristensen, K., Jensen, E.M. and Noer, I., The reflex dystrophy syndrome response to treatment with systemic corticosteroids, Acta chir. scand., 148 (1982) 653-655.

3 Davidoff, G., Roth, E., Guarracini, M., Sliwa, J. and Yarkony, G., Function-limiting dysesthetic pain syndrome among traumatic spinal cord injury patients: a cross-sectional study, Pain, 29 (1987) 39-48. 
4 Davis, S.W., Petullar, C.R., Eichberg, R.O. and Cho, D.S., Shoulder hand syndrome in a hemiplegic population: a 5 year retrospective study. Arch. phys. Med. Rehab.. 58 (1977) $353-356$

5 Driessen, J.J., Van der Wirken, C., Nicholaland, J.P.A. and Crul, J.F., Clinical effects of regional intravenous guanethidine (Ismelin) in reflex sympathetic dystrophy, Acta anesth. scand., 27 (1983) 505-509.

6 Fox, D.J. and Guire, K.E., Documentation for MIDAS. Statistical Research Laboratory. University of Michigan, Ann Arbor, MI, 1976.

7 Glynn, C.J., Basedow, R.W. and Walsh, J.A., Pain relief following post-ganglionic sympathetic blockade with intravenous guanethidine, Brit. J. Anaesth., 53 (1981) 1297-1302.

8 Graham, C., Bond, S.S., Gerkovich, M.M. and Cook, M.R., Use of the McGill Pain Questionnaire in the assessment of cancer pain: replicability and consistency, Pain, 8 (1980) $377-387$

9 Grushka, M. and Sessle, B.J., Applicability of the McGill Pain Questionnaire to the differentiation of 'toothache' pain. Pain, 19 (1984) 49-57.

10 Huskinson, E.C., Visual Analog Scale. In: R. Melzack (Ed.), Pain Measurement and Assessment, Raven Press, New York, 1983, pp. 33-37.

11 Kleinert. H.E., Cole, N.M., Wayne, L., Harvey, R., Katz, J.E. and Atasoy, E., Post-traumatic sympathetic dystrophy in hemiplegia, Orthop. Clin. N.Amer., 4 (1973) 917926.

12 Kozin, F., Genant, H.K., Bekerman, C. and McCarty, D.J., The reflex sympathetic dystrophy syndrome. II. Roentgenographic and scintigraphic evidence of bilaterality and of periarticular accentuation, Amer. J. Med.. 60 (1976) $332-338$

13 Kozin, F., McCarty, D.J., Sims, J. and Genant, H., The reflex sympathetic dystrophy syndrome. I. Clinical and histologic studies: evidence for bilaterality, response to corticosteroids and articular involvement, Amer. J. Med., 60 (1976) 321-331.

14 Kozin, F., Ryan, L.M., Carerra, G.F., Soin, J.S. and Wortmann, R.L., The reflex sympathetic dystrophy syndrome. III. Scintigraphic studies, further evidence for the therapeutic evidence of systemic corticosteroids, and proposed diagnostic criteria, Amer. J. Med., 70 (1981) 23-30.

15 Mclzack, R., The McGill Pain Questionnairc: major properties and scoring methods, Pain, 1 (1975) 277-299.

16 Melzack, R., Taenzer, P., Feldman, P. and Kirch, R.A.. Labour is still painful after prepared childbirth training, Canad. med. Ass. J., 125 (1981) 357-363.

17 Pak, T.J., Martin, G.M., Magness, J.L. and Kavanaugh. G.J. Reflex sympathetic dystrophy: review of 140 cases Minn. Med., 53 (1970) 507-512.

18 Poplawski, 7..I.. Wiley, A.M. and Muñoz, J.F., Post-traumatic dystrophy of the extremities. J. Bone Jt Surg., 65-S (1983) 642-655.

19 Prieto, E.J., Hopson, L., Bradley, L.A., Byrnc, M., Geisinger. K.F., Midax, D. and Marchisello, P.J., The language of low back pain: factor structure of the McGill Pain Questionnaire, Pain, 8 (1980) 11-19.

20 Subbarao, J. and Stillwell, G.K., Reflex sympathetic dystrophy syndrome of the upper extremity: Analysis of total outcome of management of 125 cases. Arch. phys. Med. Rehab., 62 (1981) 659-664.

21 Van Buren, I. and Kleinknecht, R.A., An evaluation of the McGill Pain Questionnaire for use in dental pain assessment, Pain, 6 (1979) 23-33.

22 Wang, J.K., Johnson, K.A. and IIstrup, D.M., Sympathetic blocks for reflex sympathetic dystrophy, Pain, 23 (1985) $13-17$. 